\title{
CLARITY techniques based tissue clearing: types and differences
}

\author{
Z. Guo ${ }^{1}$, Y. Zheng ${ }^{2}$, Y. Zhang ${ }^{1}$ \\ ${ }^{1}$ Department of General Surgery, Hepatic-biliary-pancreatic Institute, Lanzhou University Second Hospital, \\ Lanzhou University, Lanzhou, China \\ 2Department of Plastic Surgery, Lanzhou University Second Hospital, Lanzhou University, Lanzhou, China
}

[Received: 19 November 2020; Accepted: 8 January 2021; Early publication date: 9 February 2021]

\begin{abstract}
CLARITY is a tissue imaging technique that uses hydrogel embedded tissue to remove lipids while maintaining the intactness of protein and tissue fine structure. CLARITY has been widely used in the field of three-dimensional reconstruction of intact tissues and biomolecular information analysis, which enhances the ability to obtain biological structural and molecular information from intact systems. Therefore, many modified tissue clearing methods based on CLARITY have emerged. However, the variety and complexity of modified CLARITY techniques, as well as such challenges as low tissue clearing efficiency, tissue damage, and expensive experimental equipment significantly limited popular application. This review systematically summarises the progress of CLARITY techniques from the perspective of tissue clearing and classifies them into active CLARITY, passive CLARITY, and the method of merging active CLARITY with passive CLARITY according to different tissue clearing methods, which helps researchers to select a suitable tissue clearing method for the experimental samples more quickly and effectively based on balancing the removal speed and tissue transparency of different tissue clearing methods. In addition, combing through the advantage and highlighting the limitations of CLARITY techniques may be beneficial for the ideas building of different research and enlighten to improve the details of the techniques. (Folia Morphol 2022; 81, 1: 1-12)
\end{abstract}

Key words: CLARITY, three-dimensional imaging, tissue clearing, lipid removal, electrophoresis, brain, histology

\section{INTRODUCTION}

Biological tissue is a three-dimensional (3D) structure with complex detailed structures and vast molecular information, and how to acquire these data has always been a critical challenge [14, 20, 28, 35, 37, $43,44,54]$. This task has mainly relied on cutting the tissue into thin slices and providing cellular information at the 3D level. Such conventional tissue imaging inevitably loses crucial molecular information after tissue slicing and also artificially segment interconnection and function of tissues, which is particularly important for neurological studies. Therefore, how to transform intact tissues and organs into optically transparent structures has attracted people's attention. Tissue optical clearing, a unique large volume imaging method, enable tissue structures to be visualized in the whole tissue or in the whole body that has not been sliced. Tissue clearing methods achieve

Address for correspondence: Y. Zhang, PhD, MD, Department of General Surgery, Hepatic-biliary-pancreatic Institute, Lanzhou University Second Hospital, Lanzhou 730030, Gansu, China, tel. +86 18809449788, e-mail: zhangyouchengphd@163.com

This article is available in open access under Creative Common Attribution-Non-Commercial-No Derivatives 4.0 International (CC BY-NC-ND 4.0) license, allowing to download articles and share them with others as long as they credit the authors and the publisher, but without permission to change them in any way or use them commercially. 
deeper imaging in large volumes by reducing scattering and increasing the depth of light penetration $[29,45-47,53]$. Furthermore, these methods can adapt to various optical imaging techniques and show great potential to acquire 3D high-resolution images of intact tissue [5, 32].

At present, there are mainly three types of tissue clearing methods: hydrophobic, hydrophilic and hydrogel methods [39, 41]. Hydrophobic tissue clearing methods use organic solvents to achieve rapid transparency of intact tissue by reducing light scattering [44]. 3DISCO, a hydrophobic tissue clearing method, can completely clear the intact adult mouse brain within 2 days. However, the storage time of samples is short owing to fluorescence quenching $[10,11]$. The hydrophilic tissue clearing methods are carried out by using water-soluble reagents. Although the tissue clearing ability of hydrophilic tissue clearing methods (such as Scale, Scales, SeeDB, CUBIC, etc.) was sometimes inferior to that of hydrophobic tissue clearing methods, they offer unique advantages, including preservation of protein function and high levels of biosafety and biocompatibility [16, 17, 19, 38]. The hydrogel-based tissue clearing method is similar to the hydrophilic tissue clearing method, but the lipids are extracted from the hydrogel-tissue hybrid in a more aggressive way.

CLARITY (Clear Lipid-exchanged Acrylamide-hybridised Rigid Imaging/Immunostaining/In situ hybridisation-compatible Tissue-hYdrogel) proposed in 2013 is a hydrogel-based tissue clearing method, and its necessary steps are as follows: tissue fixation, hydrogel formation, lipid extraction, staining, and imaging [3]. By crosslinking acrylamide with intact tissue to form a reticular hydrogel structure, this technique provides a physical framework for tissue without destroying biomolecules and fine structure characteristics of removing lipids from intact tissue to turn it into optically transparent and permeable transparent tissue. Therefore, it can be stained and de-stained for multiple rounds without tissue sections, and then the 3D structure of the tissue can be observed and constructed under an optical microscope. CLARITY was initially applied to construct the structure of brain tissue, showing neurotransmitters, cellular relationships, subcellular structures intact-tissue imaging of long-range projections, local circuit wiring, etc. It has been widely used in the basic research of the pathological structure of nervous system-related diseases, transparent processing of intact organs (such as pancreas, lung, intestine, liver, and kidney), the relationship between organ structure and function and dynamic changes of blood vessels, 3D imaging of plant organs, 3D biogeography and metabolic status of microorganisms and other scientific research $[1,3,4,12,25,31,36]$. Furthermore, CLARITY can also be combined with traditional imaging techniques, which brings new opportunities for the fundamental study of disease pathology [36].

However, the variety and complexity of the improved techniques for CLARITY, as well as such challenges as complicated steps, low tissue clearing efficiency, tissue damage, and expensive experimental equipment significantly limited popular application. This review systematically summarises the research progress of CLARITY techniques from the perspective of tissue clearing. The brief schematic representation of CLARITY and its modifications is shown in Figure 1. In addition, since the lipids in the tissue affect the tissue transparency to a great extent, the focus of CLARITY is the extraction of lipids from the tissue [22]. Lipid extraction can be passive thermal diffusion, with lipids captured in the detergent micelle diffusing, or active by applying an electric field. The two different methods of lipid extraction are compared in Table 1. According to the way of lipid extraction, these modified CLARITY are classified as active CLARITY, passive CLARITY, and the method of merging active CLARITY with passive CLARITY to select the appropriate tissue clearing method more quickly and effectively based on the balance of tissue clearing speed and tissue transparency of different tissue clearing methods. Furthermore, combing through the advantages of CLARITY and highlighting limitations may be conducive to the construction of different research ideas and enlighten to improve the details of the techniques.

\section{PASSIVE CLARITY}

The diffusion barrier properties caused by the lipid bilayer of cells are related to chemical penetration and light scattering properties of the lipid-water interface, making the tissue difficult to visualise $[3,40,54]$. Therefore, lipid extraction is critical for the optical transparency of tissue. The original CLARITY used $4 \%$ sodium dodecyl sulphate (SDS) (sodium borate buffer, $\mathrm{pH}$ 8.5) at $37-50^{\circ} \mathrm{C}$ for electrophoretic tissue clearing. However, this may be difficult to implement and may lead to variability in the final tissue quality, including epitopes loss, damage to fine structure and tissue damage caused by heating. Fortunately, lipid extrac- 

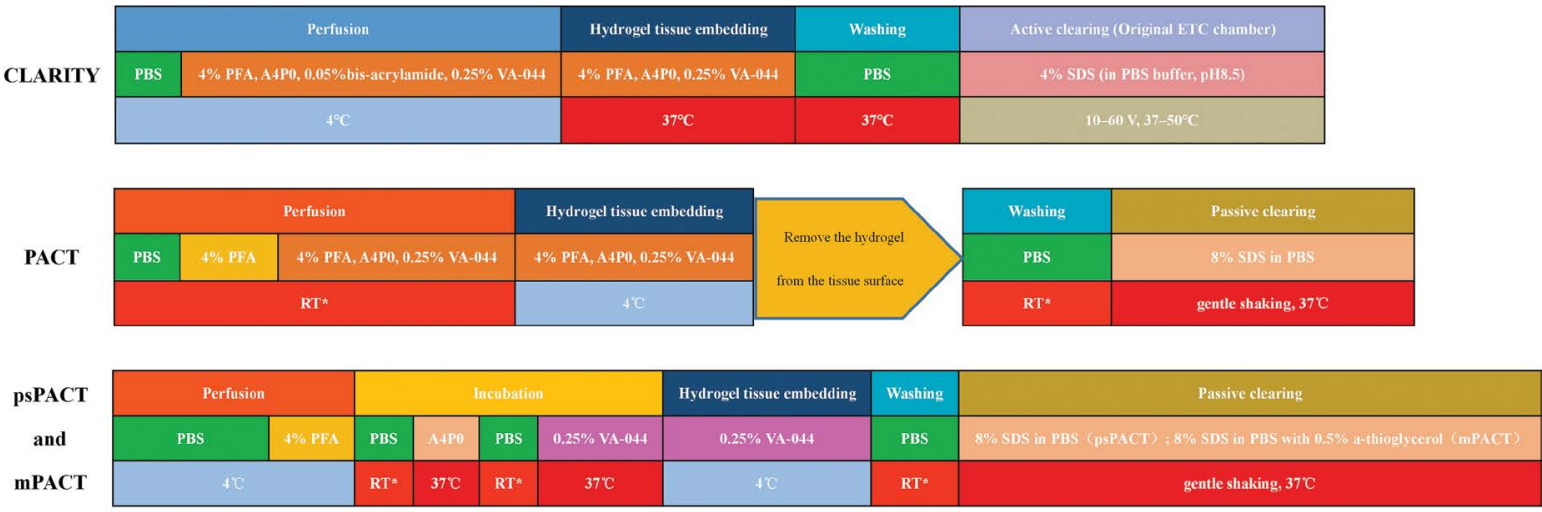

\begin{tabular}{|c|c|c|c|c|c|}
\hline \multicolumn{2}{|l|}{ Perfusion } & Fixation & Hydrogel tissue embedding & Washing & Active clearing (ACIEETC chamber) \\
\hline 0.98. NiCl contrining heparin & $4 \%$ PFA & $4 \%$ PFA & A 4 Pa, $0.25 \%$ VA-1.44 & PBS & $4 \%$ SDS and $200 \mathrm{mM}$ horic acid in $\mathrm{dH2O}(\mathrm{pH} 8.5)$ \\
\hline $\mathbf{R T}^{*}$ & & $+c$ & $37 \mathrm{C}$ & $\mathbf{R T}^{*}$ & $1.54,37 \mathrm{C}$ \\
\hline
\end{tabular}

PRE-CLARITY

\begin{tabular}{|c|c|c|c|c|c|}
\hline \multicolumn{2}{|c|}{ Perfusision } & Hydrogel tissue embedding & Washing & Active clearing (NCES) & Passive clearing \\
\hline PBS & $A 4 P 4 B 0.05$ & $\mathrm{~A} 4 \mathrm{P} 4 \mathrm{~B} 0.05$ & PBS & $4 \%$ SDS in PBS with $1 \%$ a-thioglycorol & $4 \%$ SDS in PBS with $5 \%$ atthioglycerol \\
\hline & $4 \mathrm{C}$ & $37 \mathrm{C}$ & $37^{\circ} \mathrm{C}$ & $25 \mathrm{~V}, 37 \mathrm{c}$ & gentle shaking, $50^{\circ} \mathrm{C}$ \\
\hline
\end{tabular}

Figure 1. Schematic representation of CLARITY and its modifications. An outline of the individual steps involved in each of the CLARITY and its modifications is provided alongside the reagents required for each step. Each method has three rows of boxes. The first row presents the simplified steps of the protocol; the second row shows the required reagents; the third row displays the recommended working temperature; $\mathrm{RT}$ - room temperature; other abbreviations — see text.

Table 1. Comparison of electrophoresis and passive thermal diffusion

\begin{tabular}{lcc}
\hline Methods & Electrophoresis & Passive thermal diffusion \\
\hline Clearing speed & Rapid (several hours to days) & Slow (several days to weeks) \\
Sample damage & Severe & Mild \\
The loss of biological information & More & Less \\
Cost and complexity & Expensive and difficult & Cheap and easy \\
Circulation & Need (to control the flow rate and temperature) & Needless \\
Clearing buffer & $4 \%$ SDS (in PBS buffer, pH 8.5) & $8 \%$ SDS (in PBS buffer, pH 7.5) \\
\hline
\end{tabular}

SDS — sodium dodecyl sulphate; PBS — phosphate buffered saline

tion can be passive: lipids captured in the detergent micelles can slowly diffuse out of the tissue into the detergent solution. Temperature and solution affect the rate of lipid clearing, tissue homogeneity and protein loss, but excessive temperature can also lead to tissue damage and fluorescence quenching. Tissue clearing can also accelerate the removal process by gentle shaking and constantly changing the clearing solution. Poguzhelskaya et al. proposed a simplified diffusion-based method, omitting the electrophoresis chamber, called CLARITY2 [33]. The sample (1-1.5 mm thickness) can be completely cleared by incubating it in the clearing solution for 1-2 weeks, with gentle shaking at $37^{\circ} \mathrm{C}$. Passive tissue clearing omits the time-consuming and laborious electrophoresis step and avoids the risk of tissue damage. Moreover, it was reported that there was no significant difference in protein concentration before and after between active and passive clearing [8].

However, several limitations and challenges are facing passive tissue clearing. The main weakness of passive clearing methods is the slow clearing speed, which makes them unsuitable for the clearing of large tissue volumes or whole organisms. Moreover, excessive clearing time leads to a decrease in the signal of fluorescent proteins and an increase in the loss of biomolecules. Therefore, passive CLARITY is an excellent choice for poor laboratory conditions, small samples 
(tissue thickness $<3 \mathrm{~mm}$ ), or high requirements for the integrity of tissue structure and molecular information. However, in recent years, how to promote rapid, whole-brain, and whole-body passive clearing has gradually become the focus of research, and some new modified techniques have emerged that have dramatically improved the scope of application of passive CLARITY technique.

\section{PACT and its variants}

In the original CLARITY, the experimental animals were transcardially perfused with hydrogel monomer solution (including 4\% paraformaldehyde [PFA], 4\% acrylamide, and $0.05 \%$ bis-acrylamide in phosphate buffered saline [PBS] supplemented with $0.25 \%$ VA-044 initiator). The interesting parts were selected and incubated in $4^{\circ} \mathrm{C}$ hydrogel monomer solution for 3 days and then polymerised into a hydrogel mesh [3]. Although hydrogel-tissue hybrid avoids tissue damage and the loss of biological information, the dense hydrogel mesh structure leads to low SDS transport efficiency and slow down the speed of lipid extraction. Therefore, Yang et al. [52] developed a simple version of CLARITY based on the passive lipids extraction and termed it PACT (passive clarity technique) to increase the efficiency of SDS transportation and retain the fine structure of the tissue. They perfused and fixed tissue with 4\% PFA, and then the tissue was incubated in A4P0 (4\% acrylamide in PBS supplemented with $0.25 \%$ VA-044 initiator) at $4^{\circ} \mathrm{C}$ overnight. Compared with original CLARITY, the tissue treated with A4PO increased the hydrogel mesh porosity, which significantly improves the SDS transport speed, but the tissue was also more easily to swell, and the changes of the tissue weight and volume were $174 \%$ and $223 \%$, respectively. Fortunately, the tissue will gradually shrink to the original size in the following steps, and this change of tissue volume expansion-contraction has little effect on the tissue structure, even providing an advantage for the application of some dense tissues, such as bone [15]. Moreover, since the hydrogel cross-linking inside and outside the tissue is reduced by removing bis-acrylamide from the hydrogel monomer solution during the hydrogel tissue embedding process, the tissue can simply be removed from the surrounding hydrogel, reducing the physical damage to the sample. Besides, they emphasized that increasing the concentration of the SDS clearing buffer from the original $4 \%$ to $8 \%$ can achieve uniform tissue clearing, and called
$8 \%$ SDS buffer as PACT reagents. PACT increases the efficiency of passive lipid extraction and is suitable for clearing small or particularly fragile samples and organs, which can clear 1-3 mm thick samples (including the brain, spinal cord, kidney, heart, lung and intestine, etc.) within 2-5 days.

However, PACT is still slow to clear large volumes of tissues, which limits its scope of application. The PACT reagents can be delivered either intracranially or via the vasculature to clear and label whole-brain and whole-body. A4P0 and PACT reagents were continuously circulated in the fixed animal vasculature by the peristaltic pump for tissue clearing in situ. This method, similar to cardiac perfusion, was named PARS (perfusion-assisted agent release in situ) by Yang et al. $[13,52]$. PARS increases the contact area between clearing buffer and tissue, so the rate of tissue clearing becomes faster. Furthermore, the tissue swell of PARS is reduced due to the limitation of physiological structure in the process of tissue clearing. Unfortunately, the implementation complexity of PARS limits its further application. Moreover, PACT/PARS needs more time to get maximal tissue transparency. Therefore, there are some PACT-based tissue clearing methods emerged.

Woo et al. $[49,50]$ modified PACT by treating tissues in $4 \%$ acrylamide and $0.25 \%$ VA-044 in two separate steps during hydrogel formation to improve the lipid removal speed, called psPACT (process separation PACT). This separation process avoids the removal of the remaining unpolymerised hydrogel monomers surround the tissue and the time required for lipid extraction of psPACT is also $10 \%$ less than that of PACT. Meanwhile, based on the psPACT, Woo et al. $[49,50]$ added $0.5 \%$ a-thioglycerol, a un-browning agent, to the PACT reagent to increase the lipid removal speed of most tissues by $25 \%$, and called this method mPACT (modified PACT). The addition of $0.5 \%$ a-thioglycerol to PACT reagent accelerated optical transparency but increased tissue fragility. Therefore, Woo et al. [48] proposed the process separation PACT-Acrylamide (psPACT-A) and modified PACT-Acrylamide (mPACT-A) protocols, which avoid increasing tissue fragility without reducing optical transparency and can be used to remove mouse embryos. In the modified methods, after the tissue was embedded in $0.25 \%$ VA-044, the sample was reincubated in the A4P0 solution to improve the firmness of hydrogel. Both psPACT-A and mPACT-A can achieve rapid optical tissue clearing within the same clearing time as the original protocols. 
PACT and its variants expanded the application of CLARITY, which can be used to achieve whole-brain and whole-body clearing and labelling. Moreover, these modified methods provide a powerful tool to study the mechanism of disease onset and progression, and can reflect detailed changes in a part of the observed sample as a whole to fully understand its biological characteristics.

\section{Free of acrylamide SDS-based tissue clearing}

The original CLARITY includes a hydrogel embedding step based on two hypotheses:

- The process of the extraction of lipids from tissues removes the lipid bilayers that are critical to cell integrity, but cell integrity is different from tissue integrity. Although the former may be mainly maintained by the plasma membrane, the tissue has mechanical strength, and its integrity is maintained by the intracellular cytoskeleton, cooperative transmembrane adhesion junctions, and extracellular junctions [18];

- The tissue is crosslinked with formaldehyde in the presence of hydrogel monomers, covalently linking tissue elements to monomers that are then polymerised into a hydrogel mesh. It forms a physical framework that prevents proteins from being removed from the tissue during SDS-micelle clearing, while lipids can be washed away [3]. However, the use of acrylamide hydrogel in CLARITY caused many problems. The process of hydrogel tissue embedded causes the tissue to swell and become more fragile with the loss of structural integrity $[3,26,30]$. Moreover, although the pores of hydrogel-tissue hybrid help to promote lipid extraction and antibody penetration, antibody penetration appears to be better in unhybridized, formaldehyde-fixed tissue [42, 52]. Therefore, Lai et al. [23] questioned whether acrylamide was cross-linked with formaldehyde-modified proteins. They believed that tissue-PFA-acrylamide was not crosslinked and that when the tissue was fully fixed, the use of hydrogel can be avoided in the process of lipid removal. Compared with the lipid removal process of SDS-micelles in hydrogel embedded tissue and hydrogel non-embedded tissue, there was no visible tissue swelling in the unembedded tissue of the hydrogel. Furthermore, protein loss in unembedded tissue only increased slightly, which was probably due to the diffusion limitation of hydrogel on protein leakage, rather than the sta- ble fixation of proteins in the framework formed by hydrogels. Therefore, as long as the tissue is well fixed, the use of acrylamide hydrogel can be omitted to simplify tissue clearing.

For these reasons, Liu et al. [27] developed an improved CLARITY named FASTClear (Free-of-acrylamide SDS-based Tissue Clearing). 4\% PFA/10\% neutral-buffered formalin fixed tissue, avoiding the use of hydrogel, is directly immersed in 4\% SDS for degreasing and at a temperature of $50^{\circ} \mathrm{C}$ to increase the removal rate. However, prolonged PFA fixation time will hinder the clearing rate and immune labelling due to excessive PFA cross-linking on the tissue [26]. Moreover, some tissues (such as spinal cord and cortical white matter) are difficult to become transparent after SDS delipidation. Fortunately, iDISCO can permeate optically opaque, PFA-fixed tissue in a cocktail of detergent to immunostain the tissue [34]. Then, the tissue can be optically transparent with organic solvents using the 3DISCO [9]. FASTClear is a simplified and rapid method of tissue clearing, which simplifies the process and adds potential additional benefits, including avoiding the use of toxic acrylamide, increasing antibody penetration, reducing tissue swelling, and increasing compatibility with other tissue treatment techniques. Furthermore, FASTClear can be applied to the imaging of tissues that are difficult to become transparent with traditional tissue clearing techniques, such as fresh spinal cord tissue. However, the FASTClear is limited to antibody labelling, and the penetration depth of the antibody is not uniform, which may be related to the local tissue structure.

Thus, Xu et al. [51] developed a rapid free of acrylamide SDS-based tissue clearing method by modifying and merging PACT and FASTClear to reduce tissue clearing time and preserve fluorescent signals. In the step of lipid extraction, FACT used 8\% SDS to remove lipids and removed the process of hydrogel perfusion and embedding from PACT to improve the speed of clearing. The staining step of FASTClear was used for reference, but the working temperature was reduced to $37^{\circ} \mathrm{C}$ and the $\mathrm{pH} 7.5$ clearing buffer was used, which could reduce the fluorescence quenching rate. The FACT is a simple and low-cost method of tissue clearing, which improves the preservation of cell structure, the penetration depth of antibody and the long-term retention of the fluorescence signal. Moreover, FACT avoids the use of hydrogel and reduces tissue swelling, which can be applied to the study of fine cytoarchitectural details such as microglia. 
Table 2. Electrophoretic tissue clearing with various protocols

\begin{tabular}{lcccc}
\hline Methods & CLARITY & Stochastic electrotransport & Active clarity technique & PRE-CLARITY (NCES) \\
\hline Electrodes & Wire-electrodes & Wire-electrodes & Platinum plate-electrodes & Wire-electrodes \\
Electric field & Static & Rotational & Static & Static \\
Clearing speed & $++(2$ weeks $)$ & $+++(3$ days $)$ & $++++(5-6 \mathrm{~h})$ & + \\
Sample damage & ++ & + & +++ & ++ \\
Complexity & ++ & +++ & Yes & + \\
Circulation & Yes & Yes & & No \\
\hline
\end{tabular}

${ }^{*}$ The time required to clear the mouse brain; NCES — non-circulation electrophoresis system

\section{ACTIVE CLARITY}

Accelerating the extraction of lipids from intact tissue is the key to obtain better tissue imaging. Passive lipid removal has been greatly improved, such as CLARITY2, PACT, PARS, mPACT and other modified methods, which greatly shorten the time of tissue clearing. However, the lipid extraction speed of passive clearing methods is still slow. Therefore, improvements in active clearing methods have been underway. In the original CLARITY, the SDS detergent can capture lipids in SDS micelles, which are negatively charged at alkaline solutions ( $\mathrm{pH} 7.5-8.5)$, so they can be removed under the action of an electric field in a customized electrophoresis chamber. The electrophoretic tissue clearing (ETC) system of the original CLARITY has four elements [3]:

- a power supply;

- an electrophoresis chamber containing electrodes and samples;

- a circulator used to provide fresh clearing solutions and to control the flow rate and temperature;

- a buffer filter to filter larger particles in the clearing solution.

Electrophoretic tissue clearing increases the lipid extraction speed by several orders of magnitude compared with passive clearing, and make larger samples (such as mouse brain) transparent in a few hours to days, rather than a few days to weeks, significantly increasing the rate of lipid extraction and reducing tissue swelling.

Although ETC enhances the speed of lipid extraction, it also faces some challenges. First, the too strong electric field of ETC will lead to the formation of bubbles, discoloration, and deposition of black particles on the surface of samples, making the hydrogel opaque, and larger bubbles may cause the hydrogel to break. Moreover, the electrophoresis chamber used by the active CLARITY is heated up due to the effect of Joule heating. Although the ETC clears the tissue more thoroughly at higher temperatures, samples tend to lose structural integrity due to high temperature, just like passive CLARITY. In addition, ETC uses a static electrostatic field to cause lipids to be extracted in one direction, resulting in uneven tissue clearing. Furthermore, expensive electrophoresis equipment and complex implementation steps limit the application of electrophoretic tissue clearing. In response to these challenges, there have been many modified methods about ETC in recent years. These methods are summarized in Table 2 and diagrams of these ETC systems are presented in Figure 2.

\section{Stochastic electrotransport}

The electric field can drive the movement of charged particles in the tissue with pores (such as hydrogel-tissue hybridisation), but if the tissue contains charged molecules, the electric field can also destroy the tissue. Moreover, the electrical properties in different parts of the tissue are not uniform, and different regions have different electrical properties, which may lead to the emergence of a concentrated electric field in the tissue. Therefore, when lipids are extracted using the static electric field (an electric field that is constant along a certain direction), different parts of the tissue are extracted to different degrees of lipids, which also results in uneven penetration of antibodies in the subsequent steps of staining [21]. The excessive electric field can cause tissue deformation or even tissue structural damage, and may cause bubbles to form inside the hydrogel, reducing the transparency of the tissue and even causing the hydrogel to rupture $[3,21]$. Therefore, ETC can accelerate lipid removal in a non-destructive way only at a low electric field, and dense or larger tissue cannot be effectively removed in the low electric field.

Kim et al. [21] proved that the rotational electric field could improve the diffusivity of charged molecules in porous samples, and there is a quadratic 


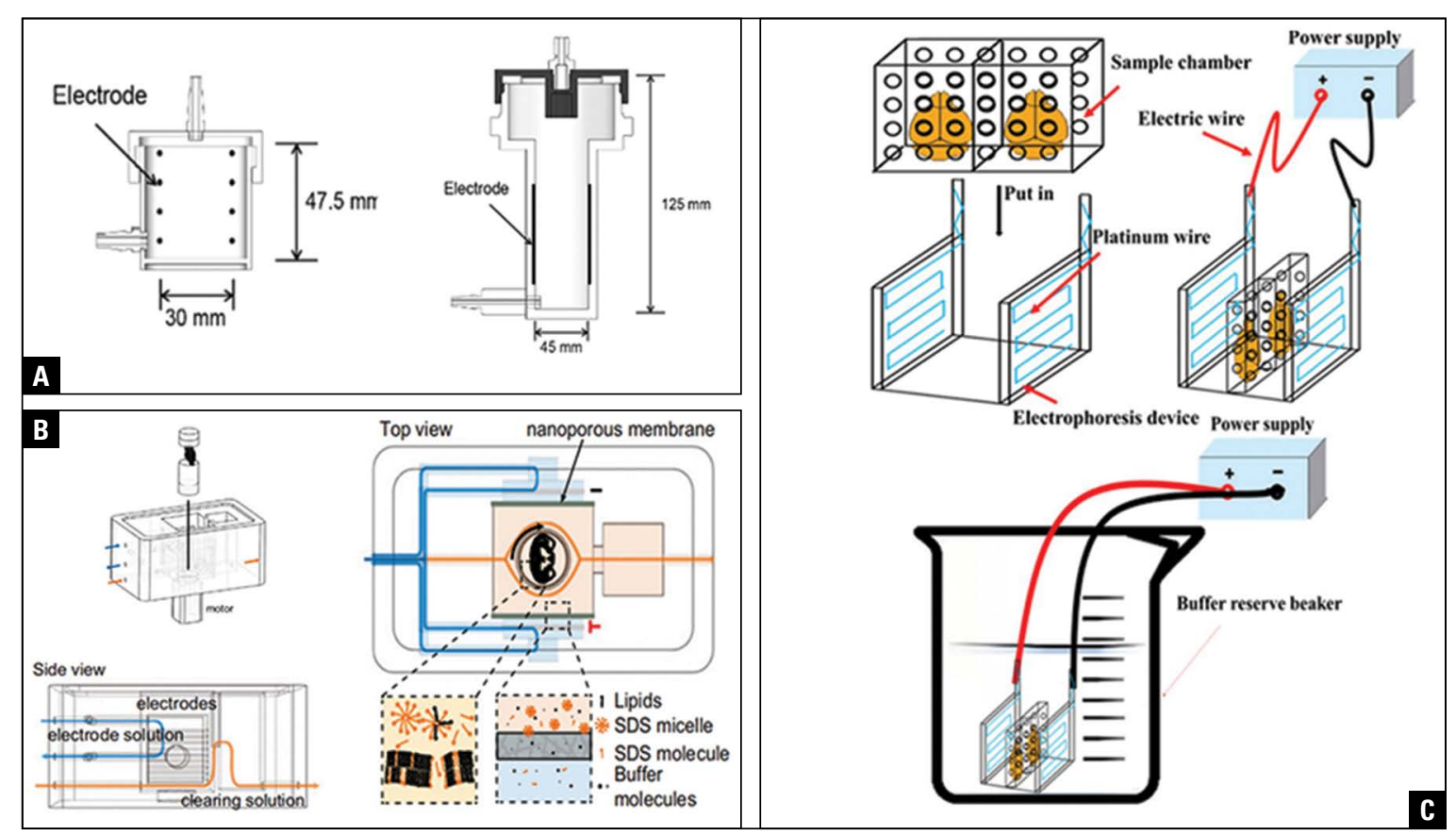

Figure 2. Comparison of electrophoretic tissue clearing (ETC) systems; A. Simplified device diagrams of the original ETC chamber (left) and ACT chamber (right), including the electrode area, electrode-electrode distance and inner-chamber dimensions. This picture is cited from the article of Lee et al. [24]; B. The device for clearing tissues using stochastic electrotransport. The sample tissue is constantly rotating relative to the electric field. This picture is cited from the article of Kim et al. [21]; C. Simplified device diagram of the non-circulation electrophoresis system (NCES). This picture is cited from the article of Du et al. [6].

correlation between the diffusivity and the electrical properties of charged molecules. Moreover, this electrophoresis-driven diffusion transport method can selectively promote the dispersion of free-moving molecules with high electromobility and inhibit the dispersion of endogenous cross-linked biomolecules with low electromobility in the samples. Therefore, Kim et al. [21] improved the method of transportation called stochastic electrotransport, which does not use the unidirectional field but uses a rotational electric field that changes the direction of the electric field over time. The rotational electric field is achieved by continuously rotating the sample chamber between the two electrodes to generate an external rotational electric field relative to the sample, which enhances the random diffusivity of charged molecules, thereby enhancing the efficiency of lipid extraction and staining of large and dense tissues with nuclear dyes, proteins, and antibodies. Moreover, stochastic electrotransport used nanoporous membranes to contain samples, preventing samples from directly contacting electrodes or electrolytic by-products. Stochastic electrotransport can completely remove the mouse organs within 1-3 days and stain them within 1 day.
The advantage of stochastic electrotransport is that it can quickly transport charged molecules out of the tissue without causing significant damage to the tissue structure. Comparing the deformation degree of tissue before and after optical clearing, there is no significant difference in the degree of tissue deformation caused by diffusion and stochastic electrotransport. On the contrary, the extraction of lipids by the static electric field may cause severe damage to the tissue, especially in the tissues with poor conductivity. Although the use of electrophoresis to remove tissue may not use an almost high voltage, the speed of electrophoresis will eventually be limited by tissue damage. Therefore, stochastic electrotransport has apparent advantages in clearing large and dense samples.

However, stochastic electrotransport has the potential for rapid lipid removal and staining, but the temperature increases with the strength of the electric field due to the effect of joule-heating. As mentioned before, the intact tissue structure and biological information will be gradually destroyed and lost as the temperature increases, which limits the further improvement of the clearing efficiency [8]. Therefore, it is significant to explore the balance point 
between the efficiency of lipid removal, temperature and electric field strength for finding the appropriate temperature and electric field strength of different tissues and organs in the following studies.

\section{Active clarity technique}

The use of electrophoresis has dramatically increased the speed of tissue clearing, but the whole process is slow and requires complex procedures. Therefore, Lee et al. [24] improved CLARITY by optimising the composition of hydrogel and electrophoresis and called this method active clarity technique (ACT). As for lipid extraction, there are two specific improvements in the ACT:

- in the process of hydrogel tissue embedding, the two-step fixation method mentioned in passive CLARITY was used [52]: 4\% PFA fixation, and then acrylamide perfusion without bisacrylamide. This method reduced the cross-linking of protein-acrylamide and obtained hydrogels with higher porosity so that lipids can be extracted quickly and staining can be better performed;

- an improved version of the ETC chamber (a dense current ETC system) was designed: the platinum plate was used to replace the thin platinum wire to produce a dense and regular current, which kept the $\mathrm{PH}$ and colour of the clearing solution unchanged during the clearing process. Moreover, the addition of an active cooling system significantly reduced the heat generation, thereby reducing the limitation of joule-heating, and can quickly obtain optically transparent tissue without causing tissue damage and protein loss. In addition, expanding the length of the ETC chamber allows all the air bubbles to float on the surface, and then all the bubbles are removed through the upper outlet of the ETC chamber, which increases the light transmittance of the hydrogel and avoids the risk that larger bubbles may cause the hydrogel to break. These improvements can quickly make large tissue samples optically transparent without causing tissue burning, collapse and protein loss.

Active clarity technique can clear most adult mouse tissues within 2-20 hours, and can expand to large organs (such as the brains of rats or rabbits) and even the whole bodies of adult animals (such as adult mouse, zebrafish and Xenopus) [24]. Comparing the clearing efficiency of ACT with other tissue clearing methods, it has been found that ACT takes 2 hours to achieve almost complete optical transparency of a $1 \mathrm{~mm}$ thick brain slice, but it takes 3 days for other methods to achieve similar optical transparency. Therefore, ACT has a great advantage in clearing thick tissues and large organs, even adult animals. The tissue cleared by ACT also showed a high contrast between connective tissue and soft tissue in PBS buffer, and the tissue structure could be distinguished even under a standard anatomical microscope. ACT, a reliable and rapid method for clearing large samples, accelerates the speed of tissue clearing and has promising applications in the field of cellular and molecular functions. However, when ACT is applied to the whole body clearing, the clearing efficiency of different tissues varies with size and density. Moreover, under conventional conditions, ACT clears some tissue with significant swelling, which requires further improvement.

\section{Fix-Clear}

The original CLARITY creatively applied hydrogel mesh to bind tissue proteins and nucleic acids to preserve the biological information of the tissue when using detergent to clear lipids. However, subsequent studies have found that hydrogel embedding is not essential for tissue clearing, and some protocols, such as SWITCH, FASTClear and FACT, use SDS for delipidation without hydrogel embedding [27, 30, 51]. Although the formation of hydrogel-tissue complexes provides lasting physical strength in the degreasing process and provides expandability, dry-ability, and the ability to selectively bind to functional chemicals, the process of hydrogel embedding has some drawbacks [7]:

- during the clearing process, the super-hydrophilic property of acrylamide causes the tissue to swell, which is not desirable for the maintenance of subcellular structures;

- crosslinking of acrylamide to proteins increases the risk of protein inactivation when immunolabelled [23, 34];

- the infiltration of acrylamide monomer into the tissue increases the complexity of the method.

Passive CLARITY first tried the method of SDS-based tissue clearing without acrylamide and made some progress. Therefore, whether this method can be applied to active CLARITY to improve tissue clearing method has attracted attention. Choi et al. [2] merged the acrylamide-free tissue clearing technique with ETC and named it Fix-Clear (FxClear). FxClear has some advantages: 
- by removal of the transcardial perfusion with hydrogel solution and hydrogel tissue embedding steps, shorter reaction time, smaller tissue expansion, and higher immunoreactivity can be achieved. Compared with ACT, FxClear provides stronger immunolabelling for most antibodies due to the absence of acrylamide;

- using a tan electrophoretic mechanism with SDS for delipidation, the lipids in the tissue can be removed quickly. However, the tissue expansion of FxClear is smaller than that in the ACT due to the absence of acrylamide, so lipids are not extracted completely, which may have a particular impact on subsequent imaging.

Furthermore, one potential problem with FxClear is that ETC can damage the tissues. Quantitative analysis of protein loss shows that ETC $>1$ day can lead to protein loss, which may be caused by tissue damage, so long-term use of ETC is not recommended. On the contrary, only a small amount $(<2 \%)$ of protein loss was detected in a safe time ( $<1$ day). FxClear is particularly suitable for the 3D pathological examination of small samples (such as brain samples of 1 2 mm). Furthermore, FxClear can be applied to some studies that require precise preservation of sample size due to the small tissue swelling.

\section{THE METHOD OF MERGING ACTIVE AND PASSIVE CLARITY}

Lipid extraction is essential for obtaining optically transparent tissue. Although electrophoresis is faster than passive thermal perfusion, it needs to establish a professional circulatory system, which makes electrophoresis difficult to manipulate. Therefore, how to design a simplified electrophoresis device to facilitate implementation has become a challenge. Du et al. [6] separates the electrode from the electrophoresis chamber and designs a mobile electrophoresis device named non-circulation electrophoresis system (NCES). Furthermore, Du et al. [6] also reported a new tissue clearing method called PRE-CLARITY (Passive pRe-electrographysics CLARITY) by merging passive thermal perfusion and electrophoretic lipid extraction for the first time. First, the A4POBO (4\% PFA in PBS) treated brain was cleared by NCES for 1 day with $4 \%$ SDS clearing buffer containing $1 \%$ alpha-thioglycerol. Then, the passive clearing was conducted in $4 \%$ SDS clearing buffer with $5 \%$ alpha-thioglycerol at $50^{\circ} \mathrm{C}$, with gentle shaking. PRE-CLARITY can make the brain
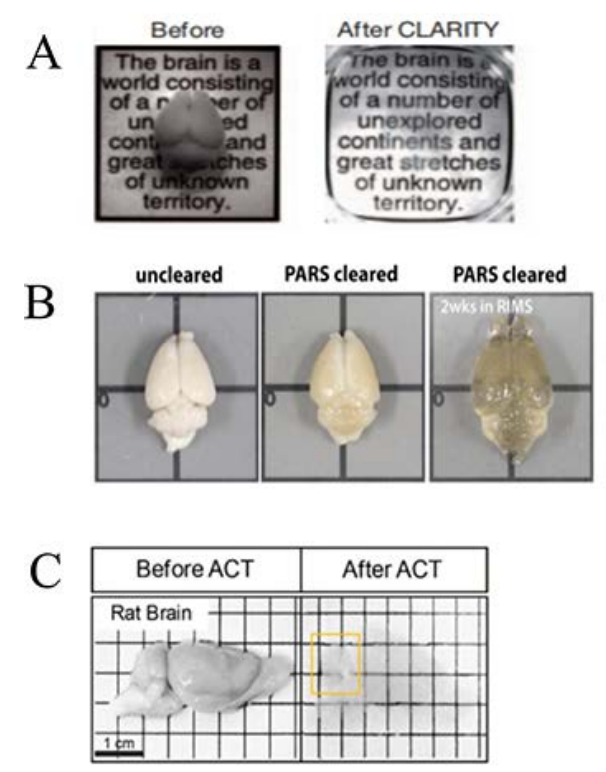

D Before clearing After electrophoresis After clearing

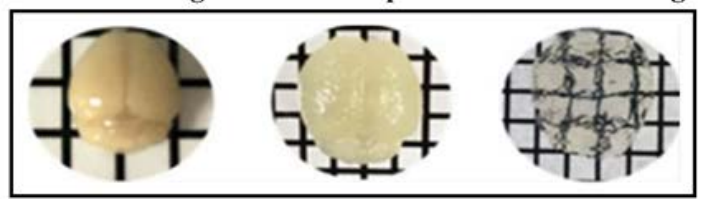

Figure 3. Brain imaging of intact adult mice and rats prepared by different methods; $\mathbf{A}$. Images of mouse brains prepared using CLARITY. The first box (from left) is the uncleared brain, and the second box is the brain processed with 8 days of CLARITY. This picture is cited from the article of Chung et al. [3]; B. Images of mouse brain before (first box, from left), after (second box) 2 weeks of PARS clearing and after immersion in RIMS (third box). This picture is cited from the article of Yang et al. [52]; C. Images of rat brain hemisphere before (first box, from left), after (second box) 15 hours of active clarity technique clearing. This picture is cited from the article of Lee et al. [24]; D. Images of mouse brains prepared using PRE-CLARITY. The first box (from left) is the A4P4B0.05-processed brain, the second box is the brain after 1 day electrophoresis at $25 \mathrm{~V} / 37^{\circ} \mathrm{C}$, and the last box is the brain after 15 days passive clearing at $50^{\circ} \mathrm{C}$. This picture is cited from the article of Du et al. [6].

completely transparent in 5 days. They had three main findings:

- when electrophoresis or passive clearing is used, A4POB0 embedding method and adding alpha-thioglycerol could achieve faster clearing and higher transparency than the A4P4B0.05 (4\% PFA, $4 \%$ acrylamide, $0.05 \%$ bis-acrylamide and $0.25 \%$ VA-044 initiator in PBS) treated brains;

- when different PFA post-fixation times are used, the cleaning speed is different. The shorter the PFA post-fixation time, the faster the clearing speed, but when using a post-fixation time less than 
10 hours, the sample is more likely to be damaged in the electrophoresis.

- compared with electrophoresis or passive clearing alone, PRE-CLARITY improved the transparency of tissue. It showed improvements in transparency over electrophoresis clearing and shortened the clearing time to half compared with passive tissue clearing (Fig. 3).

However, PRE-CLARITY also has some challenges. A4P4B0.05 treated sample was not well cleared in NCES, which may be related to the short electrophoresis time. It may also be related to the flow-assisted dissociation of lipid molecules because the main difference between the original electrophoresis system and the NCES system lies in the flow of clearing buffer. Moreover, NCES uses platinum wire to make electrodes. Platinum plates are reported to generate denser currents, thus speeding up tissue clearing, which can be improved in follow-up research. In short, PRE-CLARITY provides a simplified electrophoresis system and a faster and more transparent method of tissue clearing. The attempt to merge different tissue clearing methods also provides a new perspective for tissue optical clearing method and facilitates the development of tissue clearing method.

\section{CONCLUSIONS}

With the deepening of biomolecular information research, CLARITY and its variants will be increasingly applied to fine structure imaging of tissues and the function of cells or biomolecules in intact tissues, which has a broad application prospect. A list of variants of the CLARITY simplify and improve the experimental conditions, equipment, and concrete steps of the original CLARITY. By classifying these modified CLARITY techniques and comparing the similarities and differences between them, it is easier to find the areas where these protocols need to be improved, and we can also learn some improved ideas from them. For example, the hydrogel matrix for passive tissue clearing was optimised to the A4P0 in the PACT protocol, but this formula was later used in active tissue clearing methods. These modified methods increase the speed of tissue clearing and tissue transparency and maintain the fine structure of tissue, which is helpful to the study of different tissue types. Moreover, these improvements also break the limitation of original CLARITY. Optical clearing of intact tissues can be carried out even in ordinary laboratories, making it easier for researchers to obtain detailed biological information. Furthermore, the classification and summary of the modified CLARITY techniques will help researchers to choose a suitable tissue clearing method more quickly and effectively.

In short, passive CLARITY is a right choice for poor laboratory conditions, small samples (tissue thickness $<3 \mathrm{~mm}$ ), or high requirements for the integrity of tissue structure and molecular information. Of course, passive CLARITY can also be used to clear large samples or even whole mice, but it takes a long time, which puts forward higher requirements for the preservation of tissue and fluorescent staining. The use of active CLARITY can accelerate this process, but the high requirements of laboratory equipment limit its application. In addition, although many improvements have been made to ETC, the tissue damage caused by the current is still the main factor limiting its application. The suitable electric field intensity and electrophoresis time should be strictly selected for different texture tissues.

For the improvement of active CLARITY, the electric field conditions have been optimised in the past, whether active CLARITY can be optimised from its power source. At present, the power that drives lipids to extract from the tissue quickly is electricity, whether it can be replaced with other types of power to avoid tissue damage, such as magnetic energy or hydrodynamic pressure for molecular transport or even centrifugation. Besides, whether active or passive CLARITY, as noted by the researchers who initially developed CLARITY, the subsequent acquisition of high-resolution 3D image and how to analyse these data remains a challenge, which is a common problem in all tissue clearing methods. The improvement of image acquisition and data analysis and exploring how to combine with other technologies will not only make us more skilled in the application of CLARITY but also re-recognise the physiological characteristics of tissue or even a species as a whole for the first time from a 3D perspective.

\section{Acknowledgements}

This study was funded by the Science and Technique Innovation Project of Lanzhou University Second Hospital (CY2018-MS14), and the Doctoral Supervisors Research Fund of Lanzhou University Second Hospital (bdkyjj-04).

Conflict of interest: None declared 


\section{REFERENCES}

1. Ando K, Laborde Q, Lazar A, et al. Inside Alzheimer brain with CLARITY: senile plaques, neurofibrillary tangles and axons in 3-D. Acta Neuropathol. 2014; 128(3): 457-459, doi: 10.1007/s00401-014-1322-y, indexed in Pubmed: 25069432.

2. Choi J, Lee E, Kim JH, et al. FxClear, a free-hydrogel electrophoretic tissue clearing method for rapid de-lipidation of tissues with high preservation of immunoreactivity. Exp Neurobiol. 2019; 28(3): 436-445, doi: 10.5607/ en.2019.28.3.436, indexed in Pubmed: 31308802.

3. Chung K, Wallace J, Kim SY, et al. Structural and molecular interrogation of intact biological systems. Nature. 2013; 497(7449): 332-337, doi: 10.1038/nature12107, indexed in Pubmed: 23575631.

4. DePas WH, Starwalt-Lee R, Van Sambeek L, et al. Exposing the three-dimensional biogeography and metabolic states of pathogens in cystic fibrosis sputum via hydrogel embedding, clearing, and rRNA labeling. mBio. 2016; 7(5), doi: 10.1128/mBio.00796-16, indexed in Pubmed: 27677788.

5. Dodt HU, Leischner U, Schierloh A, et al. Ultramicroscopy: three-dimensional visualization of neuronal networks in the whole mouse brain. Nat Methods. 2007; 4(4): 331-336, doi: 10.1038/nmeth1036, indexed in Pubmed: 17384643 .

6. Du H, Hou P, Wang L, et al. Modified CLARITY achieving faster and better intact mouse brain clearing and immunostaining. Sci Rep. 2019; 9(1): 10571, doi: 10.1038/ s41598-019-46814-4, indexed in Pubmed: 31332235.

7. El-Sherbiny IM, Yacoub MH. Hydrogel scaffolds for tissue engineering: Progress and challenges. Glob Cardiol Sci Pract. 2013; 2013(3): 316-342, doi: 10.5339/ gcsp.2013.38, indexed in Pubmed: 24689032.

8. Epp JR, Niibori $\mathrm{Y}$, Liz Hsiang $\mathrm{HL}$, et al. Optimization of CLARITY for clearing whole-brain and other intact organs. eNeuro. 2015; 2(3), doi: 10.1523/ENEURO.0022-15.2015, indexed in Pubmed: 26464982.

9. Ertürk $A$, Becker $K$, Jährling $N$, et al. Three-dimensional imaging of solvent-cleared organs using 3DISCO. Nat Protoc. 2012; 7(11): 1983-1995, doi: 10.1038/nprot.2012.119, indexed in Pubmed: 23060243.

10. Ertürk $A$, Bradke $F$. High-resolution imaging of entire organs by 3-dimensional imaging of solvent cleared organs (3DISCO). Exp Neurol. 2013; 242: 57-64, doi: 10.1016/j. expneurol.2012.10.018, indexed in Pubmed: 23124097.

11. Ertürk $A$, Mauch $C P$, Hellal $F$, et al. Three-dimensional imaging of the unsectioned adult spinal cord to assess axon regeneration and glial responses after injury. Nat Med. 2011; 18(1): 166-171, doi: 10.1038/nm.2600, indexed in Pubmed: 22198277.

12. Feng Yi, Cui P, Lu X, et al. CLARITY reveals dynamics of ovarian follicular architecture and vasculature in three-dimensions. Sci Rep. 2017; 7: 44810, doi: 10.1038/srep44810, indexed in Pubmed: 28333125.

13. Gage GJ, Kipke DR, Shain W. Whole animal perfusion fixation for rodents. J Vis Exp. 2012(65), doi: 10.3791/3564, indexed in Pubmed: 22871843.

14. Gradinaru V, Treweek J, Overton K, et al. Hydrogel-Tissue chemistry: principles and applications. Annu Rev
Biophys. 2018; 47: 355-376, doi: 10.1146/annurev-biophys-070317-032905, indexed in Pubmed: 29792820.

15. Greenbaum A, Chan KY, Dobreva T, et al. Bone CLARITY: Clearing, imaging, and computational analysis of osteoprogenitors within intact bone marrow. Sci Transl Med. 2017; 9(387), doi: 10.1126/scitranslmed.aah6518, indexed in Pubmed: 28446689.

16. Hama H, Hioki H, Namiki K, et al. ScaleS: an optical clearing palette for biological imaging. Nat Neurosci. 2015; 18(10): 1518-1529, doi: 10.1038/nn.4107, indexed in Pubmed: 26368944.

17. Hama H, Kurokawa $H$, Kawano $H$, et al. Scale: a chemical approach for fluorescence imaging and reconstruction of transparent mouse brain. Nat Neurosci. 2011; 14(11): 1481-1488, doi: 10.1038/nn.2928, indexed in Pubmed: 21878933.

18. Humphrey JD, Dufresne ER, Schwartz MA. Mechanotransduction and extracellular matrix homeostasis. Nat Rev Mol Cell Biol. 2014; 15(12): 802-812, doi: 10.1038/nrm3896, indexed in Pubmed: 25355505.

19. Ke MT, Fujimoto S, Imai T. SeeDB: a simple and morphology-preserving optical clearing agent for neuronal circuit reconstruction. Nat Neurosci. 2013; 16(8): 1154-1161, doi: 10.1038/nn.3447, indexed in Pubmed: 23792946.

20. Kim JH, Jang MJ, Choi J, et al. Optimizing tissue-clearing conditions based on analysis of the critical factors affecting tissue-clearing procedures. Sci Rep. 2018; 8(1): 12815, doi: 10.1038/s41598-018-31153-7, indexed in Pubmed: 30143733.

21. Kim SY, Cho JH, Murray E, et al. Stochastic electrotransport selectively enhances the transport of highly electromobile molecules. Proc Natl Acad Sci U S A. 2015; 112(46): E6274-E6283, doi: 10.1073/pnas.1510133112, indexed in Pubmed: 26578787.

22. Kim SY, Chung K, Deisseroth K. Light microscopy mapping of connections in the intact brain. Trends Cogn Sci. 2013; 17(12): 596-599, doi: 10.1016/j.tics.2013.10.005, indexed in Pubmed: 24210964.

23. Lai HM, Liu AK, Ng WL, et al. Rationalisation and validation of an acrylamide-free procedure in three-dimensional histological imaging. PLoS One. 2016; 11(6): e0158628, doi: 10.1371/journal. pone.0158628, indexed in Pubmed: 27359336.

24. Lee E, Choi J, Jo Y, et al. ACT-PRESTO: Rapid and consistent tissue clearing and labeling method for 3-dimensional (3D) imaging. Sci Rep. 2016; 6: 18631, doi: 10.1038/srep18631, indexed in Pubmed: 26750588.

25. Lee $\mathrm{H}$, Park JH, Seo I, et al. Improved application of the electrophoretic tissue clearing technology, CLARITY, to intact solid organs including brain, pancreas, liver, kidney, lung, and intestine. BMC Dev Biol. 2014; 14: 48, doi: 10.1186/s12861-014-0048-3, indexed in Pubmed: 25528649 .

26. Liu AKL, Hurry MED, Ng OTW, et al. Bringing CLARITY to the human brain: visualization of Lewy pathology in three dimensions. Neuropathol Appl Neurobiol. 2016; 42(6): 573-587, doi: 10.1111/nan.12293, indexed in Pubmed: 26526972.

27. Liu AKL, Lai HM, Chang RCC, et al. Free of acrylamide sodium dodecyl sulphate (SDS)-based tissue clearing (FASTClear): a novel protocol of tissue clearing for 
three-dimensional visualization of human brain tissues. Neuropathol Appl Neurobiol. 2017; 43(4): 346-351, doi: 10.1111/nan.12361, indexed in Pubmed: 27627784.

28. Mano T, Albanese A, Dodt HU, et al. Whole-Brain analysis of cells and circuits by tissue clearing and light-sheet microscopy. J Neurosci. 2018; 38(44): 9330-9337, doi: 10.1523/JNEUROSCI.1677-18.2018, indexed in Pubmed: 30381424.

29. Mao Z, Zhu D, Hu Y, et al. Influence of alcohols on the optical clearing effect of skin in vitro. J Biomed Opt. 2008; 13(2): 021104, doi: 10.1117/1.2892684, indexed in Pubmed: 18465953.

30. Murray E, Cho JH, Goodwin D, et al. Simple, scalable proteomic imaging for high-dimensional profiling of intact systems. Cell. 2015; 163(6): 1500-1514, doi: 10.1016/j. cell.2015.11.025, indexed in Pubmed: 26638076.

31. Palmer WM, Martin AP, Flynn JR, et al. PEA-CLARITY: 3D molecular imaging of whole plant organs. Sci Rep. 2015; 5: 13492, doi: 10.1038/srep13492, indexed in Pubmed: 26328508.

32. Parra SG, Chia TH, Zinter JP, et al. Multiphoton microscopy of cleared mouse organs. J Biomed Opt. 2010; 15(3): 036017, doi: 10.1117/1.3454391, indexed in Pubmed: 20615019.

33. Poguzhelskaya E, Artamonov D, Bolshakova A, et al. Simplified method to perform CLARITY imaging. Mol Neurodegener. 2014; 9: 19, doi: 10.1186/1750-1326-9-19, indexed in Pubmed: 24885504.

34. Renier N, Wu Z, Simon DJ, et al. iDISCO: a simple, rapid method to immunolabel large tissue samples for volume imaging. Cell. 2014; 159(4): 896-910, doi: 10.1016/j. cell.2014.10.010, indexed in Pubmed: 25417164.

35. Rocha MD, Düring DN, Bethge $P$, et al. Tissue clearing and light sheet microscopy: imaging the unsectioned adult zebra finch brain at cellular resolution. Front Neuroanat. 2019; 13: 13, doi: 10.3389/fnana.2019.00013, indexed in Pubmed: 30837847.

36. Spence RD, Kurth $\mathrm{F}$, Itoh $\mathrm{N}$, et al. Bringing CLARITY to gray matter atrophy. Neuroimage. 2014; 101: 625-632, doi: 10.1016/j.neuroimage.2014.07.017, indexed in Pubmed: 25038439.

37. Sung K, Ding Y, Ma J, et al. Simplified three-dimensional tissue clearing and incorporation of colorimetric phenotyping. Sci Rep. 2016; 6: 30736, doi: 10.1038/srep30736, indexed in Pubmed: 27498769.

38. Susaki EA, Tainaka K, Perrin D, et al. Whole-brain imaging with single-cell resolution using chemical cocktails and computational analysis. Cell. 2014; 157(3): 726-739, doi: 10.1016/j.cell.2014.03.042, indexed in Pubmed: 24746791.

39. Susaki EA, Ueda HR. Whole-body and whole-organ clearing and imaging techniques with single-cell resolution: toward organism-level systems biology in mammals. Cell Chem Biol. 2016; 23(1): 137-157, doi: 10.1016/j.chembiol.2015.11.009, indexed in Pubmed: 26933741.

40. Syková E, Nicholson C. Diffusion in brain extracellular space. Physiol Rev. 2008; 88(4): 1277-1340, doi: 10.1152/ physrev.00027.2007.

41. Tainaka K, Kuno A, Kubota SI, et al. Chemical principles in tissue clearing and staining protocols for whole-body cell profiling. Annu Rev Cell Dev Biol. 2016; 32: 713-741, doi: 10.1146/annurev-cellbio-111315-125001, indexed in Pubmed: 27298088.

42. Treweek JB, Chan KY, Flytzanis NC, et al. Whole-body tissue stabilization and selective extractions via tissue-hydrogel hybrids for high-resolution intact circuit mapping and phenotyping. Nat Protoc. 2015; 10(11): 1860-1896, doi: 10.1038 /nprot.2015.122, indexed in Pubmed: 26492141.

43. Ueda HR, Ertürk A, Chung $K$, et al. Tissue clearing and its applications in neuroscience. Nat Rev Neurosci. 2020; 21(2): 61-79, doi: 10.1038/s41583-019-0250-1, indexed in Pubmed: 31896771.

44. Vigouroux RJ, Belle M, Chédotal A. Neuroscience in the third dimension: shedding new light on the brain with tissue clearing. Mol Brain. 2017; 10(1): 33, doi: 10.1186/ s13041-017-0314-y, indexed in Pubmed: 28728585.

45. Wang J, Zhang $Y, X u T H$, et al. An innovative transparent cranial window based on skull optical clearing. Laser Physics Letters. 2012; 9(6): 469-473, doi: 10.7452/ lapl.201210017.

46. Wen X, Mao Z, Han Z, et al. In vivo skin optical clearing by glycerol solutions: mechanism. J Biophotonics. 2010; 3(1-2): 44-52, doi: 10.1002/jbio.200910080, indexed in Pubmed: 19937846.

47. Wen X, Tuchin VV, Luo Q, et al. Controling the scattering of intralipid by using optical clearing agents. Phys Med Biol. 2009; 54(22): 6917-6930, doi: 10.1088/00319155/54/22/011, indexed in Pubmed: 19887711.

48. Woo J, Kang H, Lee EY, et al. Investigation of PRDM7 and PRDM12 expression pattern during mouse embryonic development by using a modified passive clearing technique. Biochem Biophys Res Commun. 2020; 524(2): 346-353, doi: 10.1016/j.bbrc.2019.12.133, indexed in Pubmed: 32000999.

49. Woo J, Lee EY, Park HS, et al. Novel passive clearing methods for the rapid production of optical transparency in whole CNS tissue. J Vis Exp. 2018(135), doi: 10.3791/57123, indexed in Pubmed: 29806831.

50. Woo J, Lee M, Seo JM, et al. Optimization of the optical transparency of rodent tissues by modified PACT-based passive clearing. Exp Mol Med. 2016; 48(12): e274, doi: 10.1038/ emm.2016.105, indexed in Pubmed: 27909337.

51. Xu Na, Tamadon A, Liu Y, et al. Fast free-of-acrylamide clearing tissue (FACT)-an optimized new protocol for rapid, high-resolution imaging of three-dimensional brain tissue. Sci Rep. 2017; 7(1): 9895, doi: 10.1038/s41598017-10204-5, indexed in Pubmed: 28852046.

52. Yang B, Treweek JB, Kulkarni RP, et al. Single-cell phenotyping within transparent intact tissue through whole-body clearing. Cell. 2014; 158(4): 945-958, doi: 10.1016/j. cell.2014.07.017, indexed in Pubmed: 25088144.

53. Zhu D, Larin KV, Luo Q, et al. Recent progress in tissue optical clearing. Laser Photon Rev. 2013; 7(5): 732-757, doi: 10.1002/lpor.201200056, indexed in Pubmed: 24348874.

54. Zhu X, Xia Y, Wang X, et al. Optical brain imaging: a powerful tool for neuroscience. Neurosci Bull. 2017; 33(1): 95-102, doi: 10.1007/s12264-016-0053-6, indexed in Pubmed: 27535148. 